\title{
P5CR1 protein expression and the effect of gene-silencing on lung adenocarcinoma
}

\author{
Yang She ${ }^{1}$, Aiyou Mao ${ }^{1}$, Feng $\mathbf{L i}^{2}{ }^{2}$, Xiaobin Wei ${ }^{\text {Corresp. } 1}$ \\ ${ }^{1}$ Clinical Laboratory, Central South University Xiangya School of Medicine Affiliated Haikou Hospital, Haikou city, Hainan Province, China \\ 2 Clinical Laboratory, Hainan Provincial People's Hospital, Haikou city, Hainan Province, China \\ Corresponding Author: Xiaobin Wei \\ Email address: westleywei1972@163.com
}

The present study aimed to investigate the expression of pyrroline-5-carboxylate reductase 1 (P5CR1) protein in lung adenocarcinoma and paracancerous tissues and to explore the effect of silencing the encoding gene PYCRI on the proliferation, migration, invasion, and cisplatin sensitivity in lung adenocarcinoma cells, thereby providing a novel therapeutic target for the treatment of the disease. Immunohistochemistry staining was used to detect the P5CR1 protein expression in lung adenocarcinoma and paracancerous tissues, and statistical analysis evaluated the correlation between P5CR1 protein expression and gender, age, tissue part, or pathological grade. The CCK8 assay was performed to detect the proliferation and cisplatin sensitivity, while the effect of PYCR1 on the migration and invasion of lung adenocarcinoma cells was detected by scratch test and transwell chamber assay. The findings demonstrated that the P5CR1 protein expression was significantly elevated in lung adenocarcinoma tissues and correlated with the pathological grade, whereas no significant correlation was established between the protein expression and gender, age, or tissue part. Furthermore, after PYCR1 gene silencing, the proliferation and invasion were significantly suppressed, while the sensitivity to cisplatin was significantly enhanced. Therefore, it can be speculated that the PYCRI gene affects the biological behavior of lung adenocarcinoma and cisplatin resistance, serving as a potential therapeutic target for lung adenocarcinoma. 


\section{$1 \quad P 5 C R 1$ protein expression and the effect of gene-silencing on lung}

\section{2 adenocarcinoma}

3 Yang She ${ }^{1}$, Aiyou Mao ${ }^{1}$, Feng $\mathrm{Li}^{2}$, Xiaobin Wei ${ }^{1}$

$5{ }^{1}$ Clinical Laboratory, Central South University Xiangya School of Medicine Affiliated Haikou

6 Hospital, Hainan Province, China

$7 \quad 2$ Clinical Laboratory, Hainan Provincial People's Hospital, Hainan Province, China

9 Corresponding Author:

10 Xiaobin Wei ${ }^{1}$

11 People's Avenue, Haikou City, Hainan Province, 570208 ,China.

12 E-mail address: westleywei1972@163.com

\section{Abstract}

The present study aimed to investigate the expression of pyrroline-5-carboxylate reductase 1 (P5CR1) protein in lung adenocarcinoma and paracancerous tissues and to explore the effect of silencing the encoding gene PYCRI on the proliferation, migration, invasion, and cisplatin sensitivity in lung adenocarcinoma cells, thereby providing a novel therapeutic target for the treatment of the disease. Immunohistochemistry staining was used to detect the P5CR1 protein expression in lung adenocarcinoma and paracancerous tissues, and statistical analysis evaluated the correlation between P5CR1 protein expression and gender, age, tissue part, or pathological grade. The CCK8 assay was performed to detect the proliferation and cisplatin sensitivity, while the effect of PYCRI on the migration and invasion of lung adenocarcinoma cells was detected by scratch test and transwell chamber assay. The findings demonstrated that the P5CR1 protein expression was significantly elevated in lung adenocarcinoma tissues and correlated with the pathological grade, whereas no significant correlation was established between the protein expression and gender, age, or tissue part. Furthermore, after PYCRI gene silencing, the proliferation and invasion were significantly suppressed, while the sensitivity to cisplatin was significantly enhanced. Therefore, it can be speculated that the PYCR 1 gene affects the biological behavior of lung adenocarcinoma and cisplatin resistance, serving as a potential therapeutic target for lung adenocarcinoma. 
According to the Global cancer statistics 2012, lung cancer is the primary cause of cancer mortality, and non-small cell lung cancer (NSCLC) accounted for approximately 80-90\% of lung cancer deaths worldwide(Torre et al. 2015). As an important pathological type of NSCLC, the incidence of lung adenocarcinoma is increasing year by year, and has even surpassed lung squamous cell carcinoma as the most important pathological type of NSCLC(Lortet-Tieulent et al. 2014). In China, approximately 0.3 million individuals are diagnosed with lung cancer, and of these about 0.25 million succumb to mortality annually(Chen et al. 2016). Lung cancer is associated with high incidence and mortality, causing heavy burden to the society.

A majority of the lung cancer patients do not exhibit conspicuous symptoms or only have nonspecific symptoms, such as cough, which could be easily ignored. Therefore, most patients are diagnosed only during distant metastasis and are ineligible for surgery. The detection of early lung cancer is difficult. Currently, cisplatin-based combination chemotherapy is the primary therapeutic regimen for advanced NSCLC(Willers et al. 2013). However, cisplatin alone easily elicits drug resistance. According to the American Cancer Society, 90\% of the patients succumb to cancerrelated deaths due to drug resistance(Lee \& Dutta 2007). Several of studies(Bersanelli et al. 2016; Chufan et al. 2015; Pasquier 2015) have found that the mechanism underlying the tumor resistance to chemotherapeutic drugs is associated with complicated and varied cellular signaling pathways, in which, mutations in one or multiple molecules cause tumor cell invasion and drug resistance. Therefore, exploring the precise molecular mechanism underlying the physiological behavior and drug resistance of lung adenocarcinoma is imperative.

The P5CR1 protein, which is encoded by the housekeeping gene PYCR1, reduces the pyrroline-5carboxylate (P5C) to proline in the presence of NADH and regulates the tricarboxylic acid cycle, the urea cycle, and the pentose phosphate metabolic pathway(Hu et al. 2008; Phang et al. 2013) . In addition, the P5CR1 also regulates certain biological behaviors of tumor cells. PYCR1 is one of the most commonly over-expressed metabolic genes in 1981 tumor samples spanning 19 types of cancers. This suggested that the cancer cells may be addicted to high levels of PYCR1 to sustain cell growth and provide oxidative stress resistance for cell survival(Nilsson et al. 2014). In recent years, in-depth analysis of malignant melanoma, prostate cancer, breast cancer and other tumors further confirmed the importance of PYCR1 in the occurrence and development of tumors (Cai et al. 2018; Ding et al. 2017; Ye et al. 2018; Zeng et al. 2017) . Cai et al. analyzed the data regarding the expression of PYCRI in lung cancer in the Oncomine platform and found that the PYCR 1 mRNA expression was elevated in lung adenocarcinoma tissues. This study further unveiled that PYCRI could promote the proliferation of NSCLC cells and inhibit its apoptosis, which is subsequently related to the regulation of the expressions of cyclin D1, Bcl-2 and Bcl-xl in the regulation of cell cycle of PYCRI gene (Cai et al. 2018). While other studies showed that PYCRI is regulated by microRNA-488, which can activate the p38 MAPK pathway to promote the 
proliferation and apoptosis of NSCLC(Wang et al. 2019). Nonetheless, the role of PYCRI in invasion and resistance to cisplatin of lung adenocarcinoma migration, has not yet been reported.

The present study collected specimens of lung adenocarcinoma tissue and paracancerous tissue. Immunohistochemistry (IHC) staining detected the differential expression of P5CR1 protein between cancer and paracancerous tissues, and statistical analysis evaluated the correlation between P5CR1 protein levels and the relevant clinical variables. Moreover, we established PYCRl-silenced lung adenocarcinoma cells to evaluate the changes in cell proliferation, migration, invasion, cisplatin sensitivity. This study explored the role of $P Y C R l$ in the physiological behavior of lung adenocarcinoma and the potential mechanism underlying the tumor cell resistance to cisplatin, which provided novel insights into lung adenocarcinoma to formulate strategies for the treatment.

\section{Materials \& Methods}

\section{IHC detection of P5CR1 protein}

After the approval of the ethics committee of Haikou People's Hospital with approval number SC20180050 and the participant informed consent signed by the patient, we collected the lung adenocarcinoma tissues of the patients who underwent lobectomy or segmentectomy in our hospital from April 2018 to September 2018, and they had not received radiotherapy and chemotherapy before operation. The tissue sections were deparaffinized, heated in boiling $0.01 \mathrm{M}$ sodium citrate buffer ( $\mathrm{pH}$ 6.0) for $20 \mathrm{~min}$ in a microwave for antigen retrieval, and cooled to room temperature, followed by soaking in distilled water for 10 min before blocking with $10 \%$ serum in TBS buffer for $30 \mathrm{~min}$. Subsequently, the sections were probed with the primary antibodies overnight, followed by the appropriate secondary antibodies for $60 \mathrm{~min}$ at room temperature. Next, Vulcan Fast Red Chromogen Kit2 was applied for $15 \mathrm{~min}$ before ceasing the reaction. Finally, $\mathrm{DAB}$ reagent was added and incubated until the appearance of a light yellow color, followed by addition of distilled water to stop the reaction and hematoxylin staining for 30 . The stained tissue underwent dehydration by gradient concentrations of ethanol and was air dried before observation under a microscope for image analysis.

\section{Cell culture}

Lung adenocarcinoma cells NCI-H1299 and A549 were purchased from the Shanghai Cell Institute of Chinese Academy of Sciences. The cells seeded in a 6-cm tissue culture plate with 3 $\mathrm{mL}$ complete media and incubated at $37^{\circ} \mathrm{C}$ and $5 \% \mathrm{CO}_{2}$. The cells in the exponential phase of proliferation were used for subsequent experiments.

Construction of PYCR1-silencing recombinant lentivirus vector and virus infection

According to the PYCRI gene sequences in the NCBI database, the siRNA sequences were designed as follows: 5' - CCGGGAGGGTCTTCACCCACTCCTACTCGAGT AGGAGTGGGTGAAGACCCTCTTTTTG-3' and 5'-GAATTCAAAAAGAGGGTC TTCACCCACTCCTATCTCGAGTAGGAGTGGGTGAAGACC-3'， independently. The 
108 lyophilized shRNA pair sequences were solubilized in the annealing buffer and heated for 15 min 109 at $90{ }^{\circ} \mathrm{C}$ before naturally cooling to room temperature to form the double-stranded (ds) DNA. T4 110 DNA ligase was used to ligate the empty vector with annealed dsDNA, in between EcoRI and 111 AgeI. Then, the recombinant vector construct was transfected into the competent cells. Isolated 112 colonies were subjected to the identification by PCR. The identified positive colonies were 113 individually cultured for $12-16 \mathrm{~h}$ in LB media with appropriate selection antibodies at $37^{\circ} \mathrm{C}$ before 114 sequencing. The sequencing reads were aligned against the target gene sequences, and the 115 recombinant vector harboring the correct sequences were used for subsequent experiments. The 116 plasmid was extracted using the EndoFree Midi Plasmid Kit from Tiangen. Then, the empty vector 117 GV115 or recombinant vector coming was co-transfected into 293T cells with virus packaging 118 help plasmids Helper 1.0 and Helper 2.0. In 48-72 h following transfection, the viruses were 119 collected, concentrated, and purified, and the virus titter was measured by the fluorescence method. 120 Subsequently, NCI-H1299 and A549 cells were infected with one of the two virus vectors to establish the control vector-carrying or PYCRI-silencing lung adenocarcinoma cells, and the transfection efficiency of the vector control group (NC group) and PYCRl-silencing group (KD group), respectively, was measured by fluorescence microscopy.

RT-PCR

NCI-H1299 and A549 cells in the KD/NC group and non-transfected parent cells (blank control group/CON group) were collected. The total RNA of individual groups was extracted using the TRIzol reagent, followed by the estimation of RNA concentration and quality with Nanodrop 2000/2000C Spectrophotometer. Subsequently, reverse transcription to synthesize the cDNA was performed with Promega M-MLV Kit, followed by RT-PCR. PYCR1 primers were: forward 5'GGCTGCCCACAAG ATAATGGC-3'; reverse 5'-CAATGGAGCTGATGGTGACGC -3'. GAPDH primers were used to amplify the internal gene: forward 5'-TGACTTCAACAGCGACA CCCA-3'; reverse 5'-CACCCTGTTGCTGTAGCCAAA-3'. A two-step RT-PCR was performed to detect the gene expression levels in the PYCR1-silencing group in comparison with the vector control and blank control groups.

\section{Western blotting}

The cells in the logarithmic growth phase were taken, washed twice with PBS, treated twice with lysis buffer, and then were pre-cooled for cleavage. The cells were scraped off and transferred into an EP tube. After 10-15 minutes of ice lysis, the cells were broken by ultrasound (200 W 4 times, 5 seconds each time, with 2 seconds interval). The protein concentration was determined by BCA after centrifugation for 15 minutes. The protein concentration of each sample was then adjusted to $2 \mathrm{ug} / \mathrm{mu} \mathrm{L}$. Gel electrophoresis of the samples was then performed at $120 \mathrm{~mA}$ for $1 \mathrm{~h}$. After that, the protein was transferred onto the PVDF membrane by using a transfer electrophoresis device at $4^{\circ} \mathrm{C}$ and $300 \mathrm{~mA}$ for $150 \mathrm{~min}$ at constant current. The PVDF membranes were sealed at room temperature for 1 hour using TBST solution containing 5\% skimmed milk. The first antibody was diluted by blocking solution, and then incubated with the blocked PVDF membrane at room temperature for 2 hours. After that, the membrane was washed with TBST 4 times, each time for 
1478 minutes. Next, the PVDF membrane was incubated at room temperature for $1.5 \mathrm{~h}$ and then 148 washed with TBST for 4 times, each time for 8 minutes. After that, X-ray development was done 149 and then analysis.

\section{CCK8 assay to detect the proliferation}

The proliferating cells were digested with trypsin, followed by suspension in complete media. The cells were seeded at a density of 2000 cells/well in $100 \mu \mathrm{L}$ media in the triplicate/group, in a total of five 96-well plates. Starting from the following day, $10 \mu \mathrm{L} \mathrm{CCK}-8$ reagent was added into each well an incubated at 2-4 for measuring the OD value at $450 \mathrm{~nm}$ wavelength using a microplate reader.

\section{Scratch test to detect cell migration}

$5 \times 10^{4}$ cells/well in $100 \mu \mathrm{L}$ were seeded in the 96 -well plate. Upon $90 \%$ confluency, the media were replaced with that containing low concentration serum. The scratch was generated by positioning a scratch tester on the lower center of each well and gently pushing forward. Then, the cells were rinsed 2-3 times with serum-free media before culturing in low concentration serum $(0.5 \%$ FBS $)$ and photographed. Subsequently, the cells were cultured in an incubator at $37{ }^{\circ} \mathrm{C}$ and $5 \% \mathrm{CO}_{2}$ and photographed at 8 and $24 \mathrm{~h}$ under a fluorescence microscope. The migration rate was calculated based on the post-scratch images.

\section{Transwell chamber system to detect the invasion}

Cells suspensions were prepared in serum-free media at a density of $10^{5}$ cells/well. Then, the transwell chamber system was placed on a new 24-well plate, and the upper chamber was filled with $100 \mu \mathrm{L}$ serum-free media before placing it in the $37^{\circ} \mathrm{C}$ incubator for $1 \mathrm{~h}$. Subsequently, 100 $\mu \mathrm{L}$ media in the upper chamber were replaced with $100 \mu \mathrm{L}$ cell suspension in serum-free media, while the lower chamber was filled with $600 \mu \mathrm{L}$ culture media containing $30 \% \mathrm{FBS}$, followed by incubation of the whole system at $37^{\circ} \mathrm{C}$ for $24 \mathrm{~h}$. Next, the culture media was removed from the upper chamber, and the non-migratory cells in the chamber were removed using the cotton swabs. Giemsa stain was applied on the opposite surface of the membrane to stain migrated cells for 3-5 min. Finally, the chamber was soaked, rinsed several times, air-dried, and photographed under the microscope.

\section{CCK-8 assay to detect cisplatin sensitivity}

The cells were trypsinized, resuspended in complete culture media, and seeded in the 96-well plate at a density of 4000 cells/well in $100 \mu \mathrm{L}$. Then add different concentrations of the drug. Based on the study design, the drug intervention lasted for $48 \mathrm{~h}$. After that, $10 \mu \mathrm{L}$ CCK-8 reagent was added into each well for 2-4 h without refreshing the media before measuring the OD value at $450 \mathrm{~nm}$ using a microplate reader.

Statistical analysis

SPSS 21.0, Photoshop, and GraphPad Prism 5 were used for the statistical analysis of the 
183 experimental data. Quantitative data are expressed as mean $\pm \mathrm{SD}$. The independent sample t-test

184

185

186

187 was used to compare the two groups, and the analysis of variance was used to compare the variances between the groups. $\mathrm{P}<0.05$ indicated the statistically significant difference.

\section{Results}

P5CR1 protein expression in lung adenocarcinoma vs. paracancerous tissue and its correlation with clinical variables.

IHC detection demonstrated a significant increase in the expression of P5CR1 protein in 28 cases of lung adenocarcinoma tissues than that in 27 adjacent tissues $(\mathrm{P}<0.001$; Fig. 1A-B). The correlation of $\mathrm{P} 5 \mathrm{CR} 1$ protein expression with gender, age or pathological grade respectively, was analyzed by independent samples t-test; The expression level of P5CR1 protein in different lesions was analyzed by variance analysis. The results revealed that the expression of P5CR1 in lung adenocarcinoma was significantly higher in grade IIB-IIIB group than in grade IA-IIA group $(\mathrm{P}<0.001)$, but no significant correlation was detected with gender, age, or tissue part $(\mathrm{P}>0.05$; Table 1).

\section{Construction of PYCR 1-silencing of lung adenocarcinoma cells.}

Based on the PYCRI gene sequence in the NCBI database, the siRNA sequence GGGTCTTCACCCACTCCTA was designed as shRNA and integrated into the vector. The subsequent sequencing confirmed that the recombinant virus vector carried the correct sequences (Fig. 2A). The virus vector was then infected into lung adenocarcinoma NCI-H1299 and A549 cells. The transfection efficiency was detected to be $>80 \%$ using fluorescence microscopy (Fig. 2B-C). Next, the gene silencing efficiency and protein expression of NCI-H1299 and A549 cells were verified by RT-PCR and WB, respectively. Using independent sample t-test analysis, the results showed that the PYCR1 gene silencing efficiency of NCI-H1299 and A549 cells was 92.1\% and $82.4 \%$, respectively (both $\mathrm{P}<0.01$; Fig. 2D), and the expression of P5CR1 protein was significantly down-regulated when compared with the empty vector group (both $\mathrm{P}<0.01$; Fig. 2EF). Hitherto, PYCR 1-silencing lung adenocarcinoma cells were established successfully.

\section{PYCRI-silencing inhibited the cell proliferation of lung adenocarcinoma cells}

Lung adenocarcinoma NCI-H1299 and A549 cells in PYCRl-silencing/vector control/blank control groups were cultured for $24 \mathrm{~h}$, and then cell proliferation in each group was measured using CCK8 reagent for 5 consecutive days. Independent sample t-test was performed to analyze the experimental data. The results showed that the OD450 of PYCR1-silenced group was lower than that of vector control group on days 2-5 in A549 and NCI-H1299 cells (both $\mathrm{P}<0.01$; Fig. 3A-B). This indicated that PYCR 1 -silencing inhibited the proliferation of lung adenocarcinoma cells.

PYCRl-silencing has no effect on the migration ability of lung adenocarcinoma cells

Lung adenocarcinoma NCI-H1299 and A549 cells in PYCRl-silencing/vector control/blank control groups were inoculated on 96-well plate, respectively. Scratch size was measured under

Peer) reviewing PDF | (2018:12:33827:1:1:NEW 23 Mar 2019) 
219 inverted fluorescence microscope at 0,8 and 24 hours after the scratch. The width of the scratch area was determined by the $\mathrm{W}$ value derived from the Photoshop Software, which was further analyzed by independent sample t-test. The results demonstrated significant differences between the vector control group and the PYCR l-silenced lung adenocarcinoma NCI-H1299 cells after 24 $\mathrm{h}$ post-scratch $(\mathrm{P}<0.05$; Fig. $3 \mathrm{C}$-D), whereas the variation in the migration rate was $<20 \%$. This further indicated the lack of positive results. Compared to the vector controls, PYCR 1 -silencing lung adenocarcinoma A549 cells showed no significant difference in the migration ability at 24-h post-scratch ( $\mathrm{P}>0.05$; Fig. 3C-D).

\section{PYCR1-silencing suppresses the cell invasion ability of lung adenocarcinoma cells}

Lung adenocarcinoma NCI-H1299 and A549 cells in PYCRl-silencing/vector control/blank control groups were inoculated into the upper chamber of Transwell chamber. After 24 hours of incubation at $37^{\circ} \mathrm{C}$, Giemsa staining was performed to detect the number of cells migrating to the lower chamber and adhering to the bottom of the polycarbonate membrane. Independent sample t-test was used to analyze the experimental results. Transwell assay demonstrated that the number of lung adenocarcinoma NCI-H1299 and A549 cells migrating to the inferior cavity in the pycr1 silencing group was significantly reduced when compared with the vector control group (both $\mathrm{P}<0.01$; Fig. 4A-C), indicating that PYCR 1-silencing led to a significant reduction in the invasiveness of lung adenocarcinoma NCI-H1299 and A549 cells.

\section{PYCR1-silencing enhances the sensitivity of lung adenocarcinoma cells to cisplatin}

Lung adenocarcinoma NCI-H1299 and A549 cells in PYCRl-silenced/vector control/blank control groups were treated with different concentrations of cisplatin, and the rate of inhibition on the proliferation was detected using the CCK8 assay. Independent sample t-test was used to analyze the experimental results. The cisplatin stimulation with 1, 2, 4, 6, 8, 10,16mg/L significantly inhibited the NCI-H1299 and A549 cell proliferation in the PYCRI-silenced group as compared to the vector control group (both $\mathrm{P}<0.01$; Fig.4D-E). These results indicated that the PYCRIsilencing elevated cisplatin sensitivity in lung adenocarcinoma cells.

\section{Discussion}

5-pyrrolinecarboxylic acid reductases (P5CRs) are housekeeping proteins that are widely present in the prokaryotic cells as well as eukaryotic cells and contain three isoenzymes: P5CR1, P5CR2, and P5CR3. The P5CRs-proline metabolic cycle reaction is not only closely associated with the body's tricarboxylic acid cycle, urea cycle, and pentose phosphate pathway, but it also plays a regulatory role in the proliferation and apoptosis of cells, as well as, skin, bone, brain and other tissues' development and in the process of occurrence and development of oral, head and neck, lymphoma and other tumors(Liu et al. 2005; Phang et al. 2012; Reversade \& Escande 2009). The human P5CR1 gene, PYCR1 is located on chromosome 17q25. 3 and is $5 \mathrm{~kb}$ in length including 7 exons and 8 introns(Jessica et al. 2012). P5CR1 is involved in amino acid synthesis and metabolism, and promoted the occurrence and development of malignant melanoma, prostate 
256

257

258

259

260

261

262

263

264

265

266

267

268

269

270

271

272

273

274

275

276

277

278

279

280

281

282

283

284

285

286

287

288

289

290

291

292

293

294

295

296

cancer, lung cancer, breast cancer and other tumors(Cai et al. 2018; Ding et al. 2017; Ye et al. 2018; Zeng et al. 2017). Currently, some studies suggested that PYCR1 may regulate the biological behavior of tumors by regulating the transport of NADH reduction equivalent from cytoplasm to mitochondria, further affecting the level of intracellular reductant synthesis and the sensitivity to oxidative damage(Ping et al. 2009). Also P5CRs may reduce the production of reactive oxygen species in the mitochondria through oxidative respiratory chain process involved in NADP + to increase the survival rate of tumor cells(Phang et al. 2012).

The present study investigated the expression of P5CR1 protein in lung adenocarcinoma and the effect of encoding gene-silencing on the physiology of the cells. P5CR1 protein was highly expressed in lung adenocarcinoma tissues, and its level was found that to be positively correlated with the pathological grade. Thus, we speculated that the PYCRI gene might be related to the specific physiological characteristics of lung adenocarcinoma. Moreover, to probe the effect of differential expression of the gene on the proliferation, migration, invasion, and drug sensitivity of lung adenocarcinoma, we successfully established the PYCRI-silenced lung adenocarcinoma cells. Furthermore, CCK8 assay demonstrated that PYCR1-silencing significantly inhibited the cell proliferation in lung adenocarcinoma. The cell migration was detected by the scratch test, and the silencing of the PYCRI gene did not affect the migration of lung adenocarcinoma cells significantly. Next, the lung adenocarcinoma cell invasion analysis by Transwell chamber system demonstrated that PYCR 1 -silenced lung adenocarcinoma cells showed a significant reduction in the invasion ability. In order to explore the role of PYCRI in tumor cell resistance to cisplatin, lung adenocarcinoma cells in PYCR1-silenced/vector control/blank control groups were treated with cisplatin at different concentrations, followed by detection of inhibition of the proliferation using the CCK 8 assay. The results demonstrated the PYCRI-silencing led to a rise in cisplatin sensitivity in lung adenocarcinoma cells.

Although some studies explored the role of PYCR1 in the proliferation of human lung squamous cell carcinoma H1703 cells and human lung adenocarcinoma SPC-A1 cells(Cai et al. 2018), the correlation of PYCRl gene with cell migration, invasion, and cisplatin sensitivity in lung adenocarcinoma was not examined. The current study selected the lung adenocarcinoma cell lines HCI-H1299 and A549. The results demonstrated that PYCR1 promoted lung adenocarcinoma cell proliferation, which was in agreement with the above study on human lung adenocarcinoma SPCA1 cells. This study is the first to explore the effect of PYCR 1 gene on migration, invasion, and cisplatin sensitivity of lung adenocarcinoma, and confirmed that PYCRI gene promoted the invasion and caused cisplatin resistance. Although the results of the study indicated that silencing of PYCR 1 gene showed no significant effect on the migration of lung adenocarcinoma cells, while significantly promoted the invasiveness. This might be due to that the PYCRI has little effect on simple horizontal movement of lung adenocarcinoma cells, but can regulate some substances, which led to the remodeling and degradation of extracellular matrix, thereby enhancing the invasiveness of cells. PYCR1 converts the P5C into proline and increases NAD+ production. Studies have shown that elevated levels of NAD+ can promote glycolysis of lactic acid through glyceraldehyde 3-phosphate dehydrogenase and lactate dehydrogenase, resulting in increased 
297 lactic acid content(Tan et al. 2013; Yamamoto et al. 2017). Excessive lactic acid activates 298 monocarboxylic acid transporter 1 (MCT 1), causing intracellular lactic acid efflux. Extracellular 299 low $\mathrm{pH}$ value provides a good microenvironment for the release and activation of matrix 300 metalloproteinases, tumor cathepsin B and so on. Activation of these enzymes can promote 301 extracellular matrix degradation, and remodeling, leading to tumor metastasis(Dhup et al. 2012).

302 Therefore, we speculated that PYCR 1 enhanced the invasion of lung adenocarcinoma cells and this 303 might be related to its enhancement of glycolysis and lactic acid production in tumor cells. This 304 study demonstrated that silencing the PYCR1 gene can enhance the sensitivity to cisplatin that 305 rendered PYCRI as a promising candidate target for the treatment of lung adenocarcinoma. 306 However, the PYCR1-overexpression study has not been conducted and remain only at the cellular 307 level. Thus, the future experiments will study the effect of PYCRl-overexpression on the 308 physiological behavior of lung adenocarcinoma in the corresponding cellular and animal 309 experiments.

310 Considering that P5CR1 is an internal protein close to the mitochondrial inner membrane and plays 311 a key role in the regulation of substance/energy metabolism. Therefore, we consider that PYCRI 312 gene may influence the biological behavior of lung adenocarcinoma cells by regulating cell 313 energy/material metabolism, and this is also our next research direction.

\section{Conclusions}

315 This study found that the PYCRI gene is highly expressed in lung adenocarcinoma cells, and is positively correlated with the pathological grade of lung adenocarcinoma. The PYCRI gene promotes the physiological behaviors such as proliferation and invasion of lung adenocarcinoma cells. Furthermore, PYCR1 plays a critical role in resistance of lung adenocarcinoma to cisplatin, which might be a potential therapeutic target for lung adenocarcinoma, thereby providing theoretical data for the chemotherapy of the disease.

\section{Acknowledgments}

We greatly appreciate Huang Deng gao and Gao xin for experimental image processing and the Central Laboratory of Haikou People's Hospital for providing the experimental platform.

\section{References}

Bersanelli M, Minari R, Bordi P, Gnetti L, Bozzetti C, Squadrilli A, Lagrasta CA, Bottarelli L, Osipova G, Capelletto E, Mor M, and Tiseo M. 2016. L718Q Mutation as New Mechanism of Acquired Resistance to AZD9291 in EGFR-Mutated NSCLC. J Thorac Oncol 11:e121-123.

Cai F, Miao Y, Liu C, Wu T, Shen S, Su X, and Shi Y. 2018. Pyrroline-5-carboxylate reductase 1 promotes proliferation and inhibits apoptosis in non-small cell lung cancer. Oncol Lett 15:731-740.

Chen W, Zheng R, Baade PD, Zhang S, Zeng H, Bray F, Jemal A, Yu XQ, and He J. 2016. Cancer statistics in China, 2015. CA Cancer J Clin 66:115-132. 
332

333

334

335

336

337

338

339

340

341

342

343

344

345

346

347

348

349

350

351

352

353

354

355

356

357

358

359

360

361

362

363

364

365

366

367

368

369

370

371

372

Chufan EE, Sim HM, and Ambudkar SV. 2015. Molecular basis of the polyspecificity of P-glycoprotein (ABCB1): recent biochemical and structural studies. Adv Cancer Res 125:71-96.

Dhup S, Dadhich RK, Porporato PE, and Sonveaux P. 2012. Multiple biological activities of lactic acid in cancer: influences on tumor growth, angiogenesis and metastasis. Curr Pharm Des 18:1319-1330.

Ding J, Kuo ML, Su L, Xue L, Luh F, Zhang H, Wang J, Lin TG, Zhang K, Chu P, Zheng S, Liu X, and Yen Y. 2017. Human mitochondrial pyrroline-5-carboxylate reductase 1 promotes invasiveness and impacts survival in breast cancers. Carcinogenesis 38:519-531.

Hu CA, Phang JM, and Valle D. 2008. Proline metabolism in health and disease. Preface. Amino Acids 35:651-652.

Jessica DI, Boris R, Richardson AD, Scott DA, Pedro AB, De SK, Marat K, Maurizio P, Ze'Ev R, and Osterman AL. 2012. Functional specialization in proline biosynthesis of melanoma. PLoS One 7:e45190.

Lee YS, and Dutta A. 2007. The tumor suppressor microRNA let-7 represses the HMGA2 oncogene. Genes Dev 21:1025-1030.

Liu Y, Borchert GL, Donald SP, Surazynski A, Hu CA, Weydert CJ, Oberley LW, and Phang JM. 2005. MnSOD inhibits proline oxidase-induced apoptosis in colorectal cancer cells. Carcinogenesis 26:1335.

Lortet-Tieulent J, Soerjomataram I, Ferlay J, Rutherford M, Weiderpass E, and Bray F. 2014. International trends in lung cancer incidence by histological subtype: adenocarcinoma stabilizing in men but still increasing in women. Lung Cancer 84:13-22.

Nilsson R, Jain M, Madhusudhan N, Sheppard NG, Strittmatter L, Kampf C, Huang J, Asplund A, and Mootha VK. 2014. Metabolic enzyme expression highlights a key role for MTHFD2 and the mitochondrial folate pathway in cancer. Nat Commun 5:3128.

Pasquier B. 2015. SAR405, a PIK3C3/Vps34 inhibitor that prevents autophagy and synergizes with MTOR inhibition in tumor cells. Autophagy 11:725-726.

Phang JM, Liu W, and Hancock C. 2013. Bridging epigenetics and metabolism: role of non-essential amino acids. Epigenetics 8:231-236.

Phang JM, Liu W, Hancock C, and Christian KJ. 2012. The proline regulatory axis and cancer. Front Oncol 2:60.

Ping G, Irina T, Tsung-Cheng C, Yun-Sil L, Kayoko K, Takafumi O, Zeller KI, Marzo AM, De, Eyk JE, Van, and Mendell JT. 2009. c-Myc suppression of miR-23a/b enhances mitochondrial glutaminase expression and glutamine metabolism. Nature 458:762-765.

Reversade B, and Escande BNA. 2009. Mutations in PYCR1 cause cutis laxa with progeroid features. Nat Genet 41:1016.

Tan B, Young DA, Lu ZH, Wang T, Meier TI, Shepard RL, Roth K, Zhai Y, Huss K, Kuo MS, Gillig J, Parthasarathy S, Burkholder TP, Smith MC, Geeganage S, and Zhao G. 2013. Pharmacological inhibition of nicotinamide phosphoribosyltransferase (NAMPT), an enzyme essential for NAD+ biosynthesis, in human cancer cells: metabolic basis and potential clinical implications. J Biol Chem 288:3500-3511.

Torre LA, Bray F, Siegel RL, Ferlay J, Lortet-Tieulent J, and Jemal A. 2015. Global cancer statistics, 2012. CA Cancer J Clin 65:87-108.

Wang D, Wang L, Zhang Y, Yan Z, Liu L, and Chen G. 2019. PYCR1 promotes the progression of non-small-cell lung cancer under the negative regulation of miR-488. Biomed Pharmacother 111:588-595.

Willers H, Azzoli CG, Santivasi WL, and Xia F. 2013. Basic mechanisms of therapeutic resistance to radiation and chemotherapy in lung cancer. Cancer $J$ 19:200-207.

Yamamoto M, Inohara H, and Nakagawa T. 2017. Targeting metabolic pathways for head and neck cancers

Peer) reviewing PDF | (2018:12:33827:1:1:NEW 23 Mar 2019) 
therapeutics. Cancer Metastasis Rev 36:503-514.

374 Ye Y, Wu Y, and Wang J. 2018. Pyrroline-5-carboxylate reductase 1 promotes cell proliferation via inhibiting 375 apoptosis in human malignant melanoma. Cancer Manag Res 10:6399-6407.

376 Zeng T, Zhu L, Liao M, Zhuo W, Yang S, Wu W, and Wang D. 2017. Knockdown of PYCR1 inhibits cell proliferation and colony formation via cell cycle arrest and apoptosis in prostate cancer. Med Oncol 34:27. 


\section{Table 1 (on next page)}

Correlation of P5CR1 protein level with clinical variables.

$\mathrm{n}$ : number of patients; $\mathrm{P}$ : significant level; $\mathrm{R}+\mathrm{U}$ : right upper lobe; $\mathrm{R}+\mathrm{L}$ : right lower lobe; $\mathrm{L}+\mathrm{U}$ : left upper lobe; L+L: left lower lobe. 


\begin{tabular}{llll}
\hline & & \multicolumn{2}{c}{ P5CR1 protein } \\
\cline { 3 - 4 } Parameters & $\mathrm{n}$ & Expression & $P$ \\
\hline Gender & & & 0.397 \\
$\quad$ Male & 16 & $3.002 \pm 2.296$ & \\
Female & 12 & $3.753 \pm 2.266$ & \\
Age & & & 0.873 \\
$\quad \leqslant 60$ & 14 & $3.361 \pm 2.341$ & \\
$>60$ & 14 & $3.504 \pm 2.279$ & \\
Pathological grading & & & \\
I A- II A & 14 & $1.933 \pm 1.072$ & \\
II B-III B & 14 & $4.937 \pm 2.165$ & \\
Tissue part & & & \\
R+U & 8 & $4.125 \pm 2.416$ & \\
R+L & 8 & $3.125 \pm 3.091$ & \\
L+U & 6 & $3.000 \pm 1.549$ & \\
L+L & 6 & $3.333 \pm 1.633$ & \\
\hline
\end{tabular}

1

2

3

4

5

6

7

8

9

10 


\section{Figure 1}

IHC detection of P5CR1 protein expression

(A) The P5CR1 antibody binds to the P5CR1 protein in the cell pellet and is stained brown. scoring system for immunohistochemical staining: The percentage of positive cells was determined on a scale of 0-4 points, where 0 points, no positive cells; 1 point, the percentage of positive cells was less than 10\%; 2 points, the percentage of positive cells was greater than $10 \%$ but less than $50 \%$; 3 points, the percentage of positive cells was greater than $50 \%$ but less than $80 \%$; and 4 points, the percentage of positive cells is greater than $80 \%$. The staining intensity was rated on a scale of 0-3 points by negative, weak, medium and strong staining, respectively. The final score is multiplied by the two metrics; (B) The data are presented as mean and SD of P5CR1 protein in lung adenocarcinoma $(n=28)$ and adjacent tissues $(n=27) ;(* * * p<0.001)$
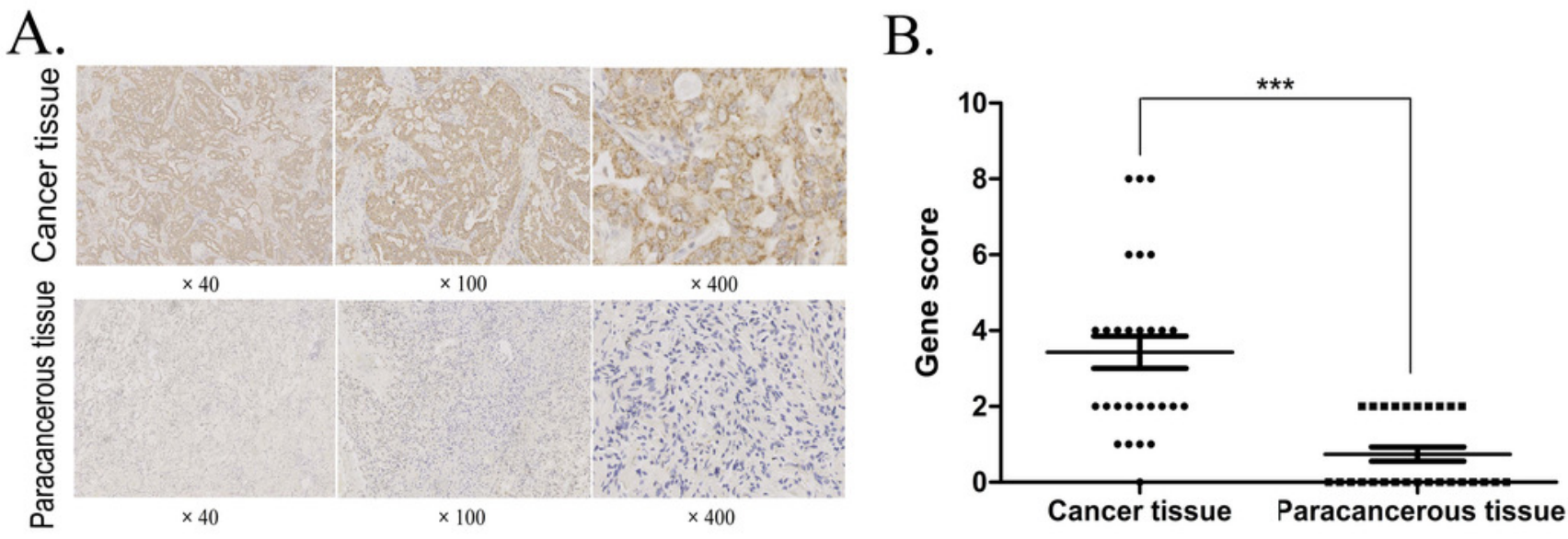


\section{Figure 2}

Construction of PYCR1-silenced lung adenocarcinoma cells

(A) Sequencing of siRNA to confirm the sequence. (B-C) Fluorescence microscopy for the detection of transfection efficiency. Positive cells presented green fluorescence at 100X magnification. (D) RT-PCR for the detection of PYCR1 expression. mRNA expression levels in KD group were significantly decreased compared with NC groups. Data are presented as mean $(n=3)$ and SD. (E) Western blotting was used to detect the expression of P5CR1 protein. (F) Histogram showed the expression of P5CR1 protein. The expression level of P5CR1 protein in KD group was significantly decreased compared with NC groups. Data are presented as mean $(n=3)$ and SD. $(* * P<0.01)$. 
A.

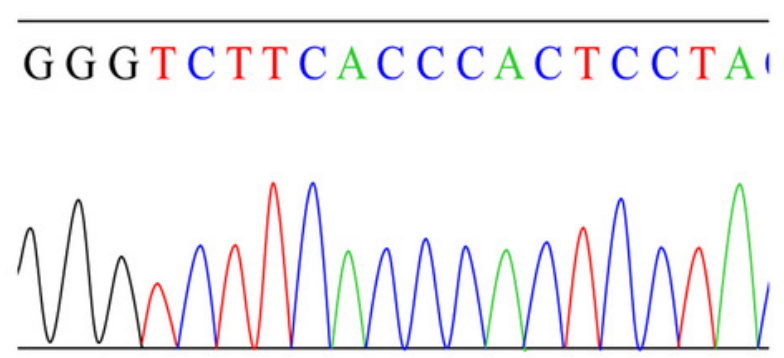

B.

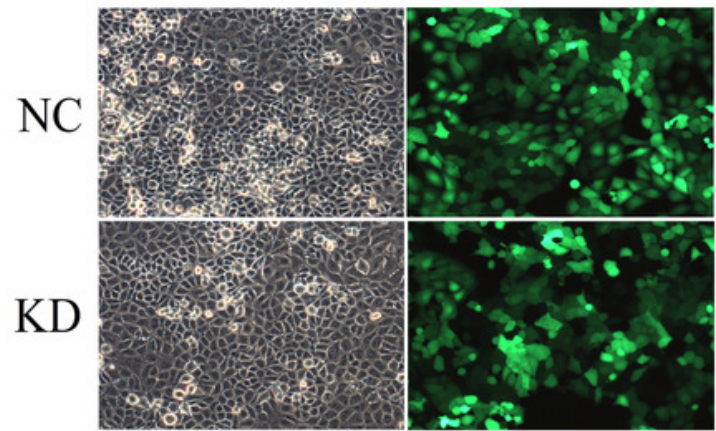

NCI-H1299
D.

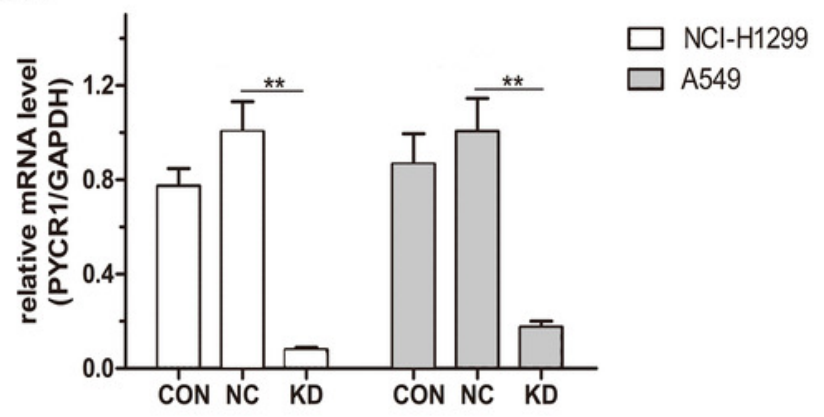

KD

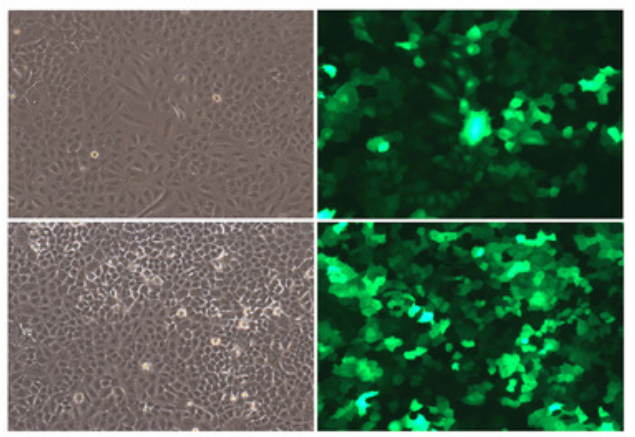

A549

$\mathrm{F}$.

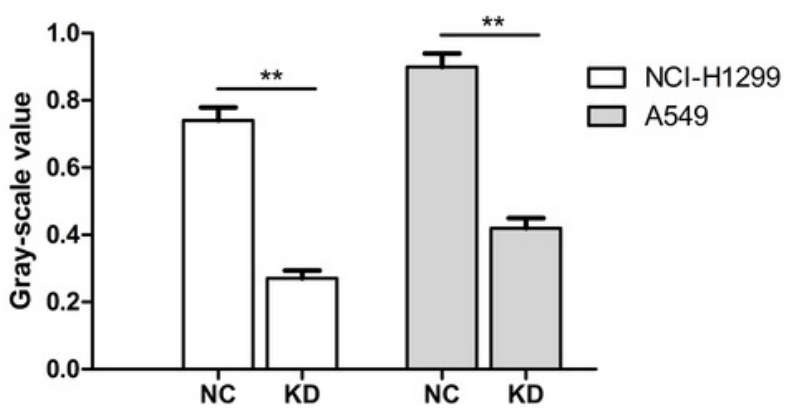




\section{Figure 3}

Detection of proliferation and migration ability of lung adenocarcinoma cells

(A-B) Three differently colored lines were used to simulate changes in the cell viability over a period in different groups of lung adenocarcinoma cells. The proliferation of the KD group was significantly inhibited compared with the NC groups. Data are represented as mean ( $n=$ 5) and SD. (C) The distances marked in the figure represent the size of the scratches measured by inverted fluorescence microscopy at 0 and $24 \mathrm{~h}$ in the three different groups of $\mathrm{NCl}-\mathrm{H} 1299$ and A549 cells. The images were captured at 40x. (D) This histogram represents the cell migration ability. PYCR1-Silencing had no effect on migration of NCl-H1299 and A549 cells in lung adenocarcinoma. Data are represented as mean $(n=3)$ and SD. $(* P<0.05 \square * * P<0.01)$. 


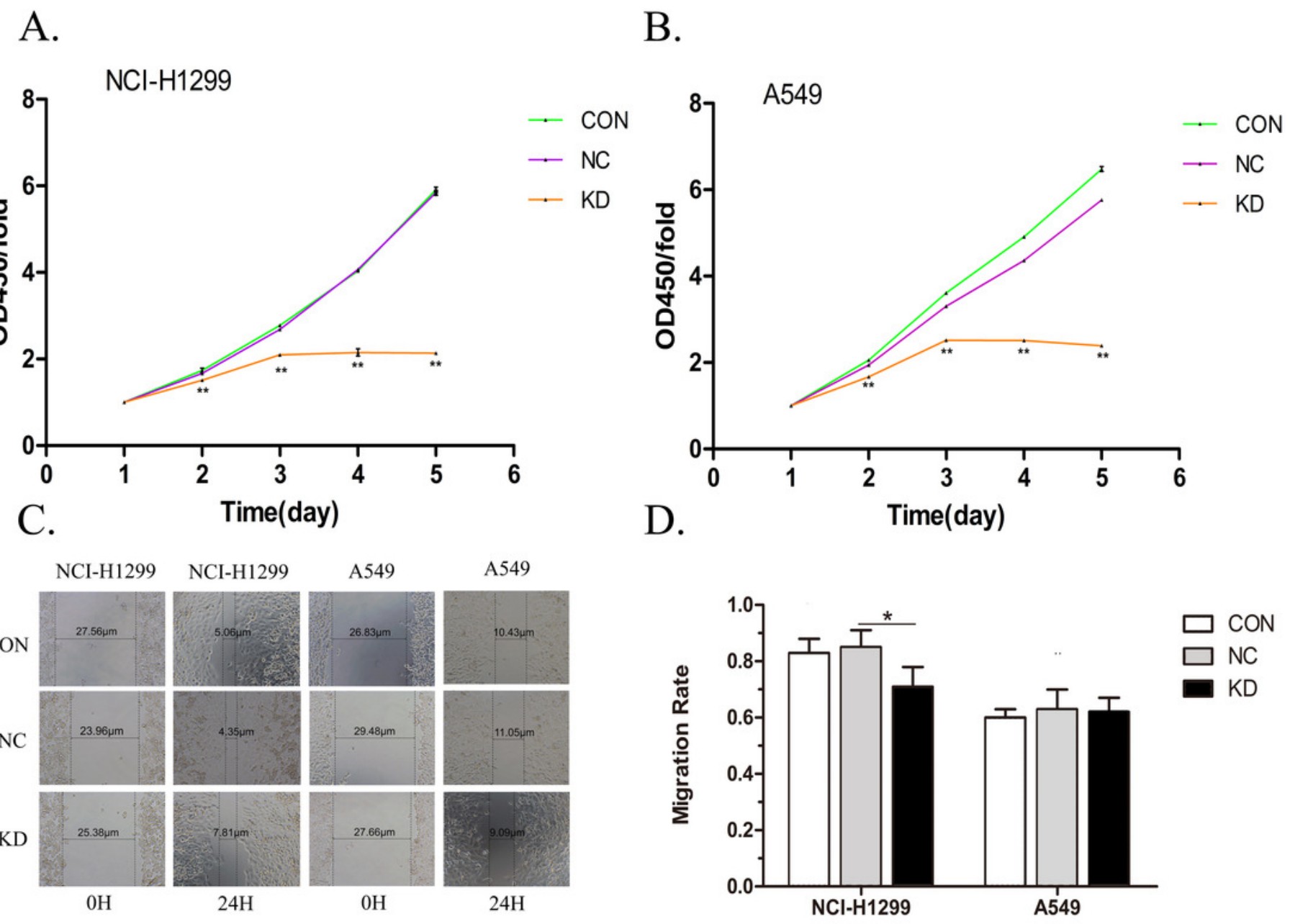




\section{Figure 4}

Detection of invasion and cisplatin resistance in lung adenocarcinoma cells

(A-B) Lung adenocarcinoma NCl-H1299 (A) and A549 (B) cells that penetrate the polycarbonate membrane in the lower chamber are stained purple. (C) This histogram represents the cell invasion ability of lung adenocarcinoma. Compared with NC groups, the number of migration to the lower chamber in KD group was significantly reduced. Data are represented as mean $(n=3)$ and SD. (D-E) Three different color lines were used to simulate the effects of different concentrations of cisplatin on cell proliferation in each group. The proliferation of the KD group was significantly inhibited compared with the NC groups. Data are represented as mean $(n=3)$ and SD. $(* P<0.05 ; * * P<0.01)$.

A.

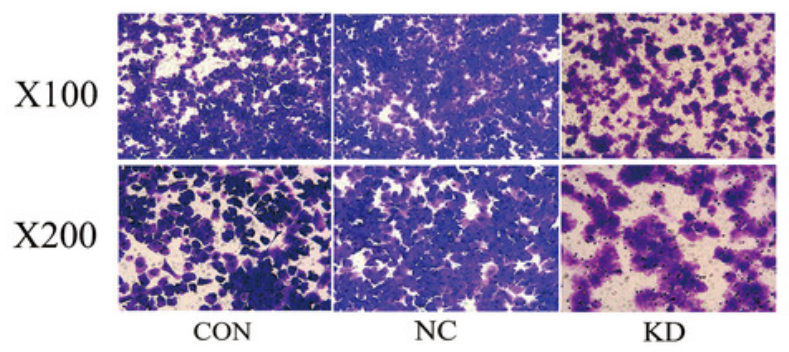

C.

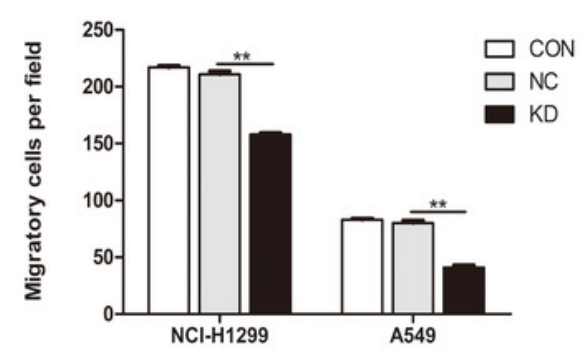

B.

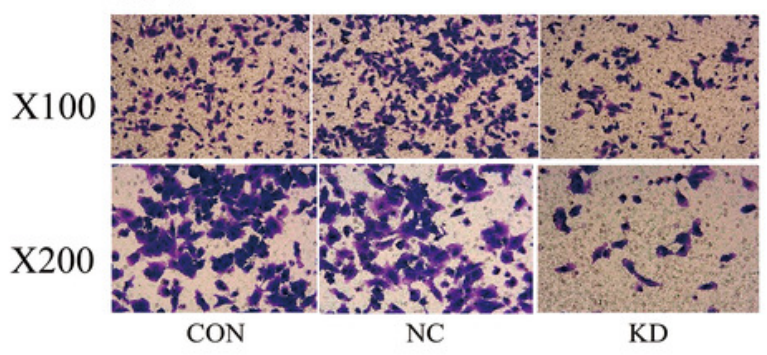

E.

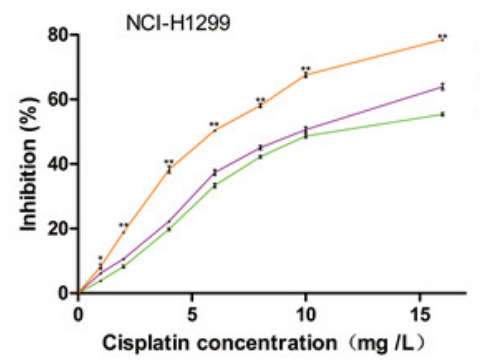

